\title{
Comparison of Two Possible Evolutionary Mechanisms of the 62 tRNA Codon Sequences
}

\author{
Fangping Wei ${ }^{*}$, Zhenxiong Lan², Luming Shen'1, Bourong Liu' ${ }^{1}$ Yu Xue ${ }^{1}$ \\ ${ }^{1}$ Physical Science \& Technology College, Guangxi University, Nanning, China \\ ${ }^{2}$ College of Computer and Information Engineering, Guangxi Teacther Education University, Nanning, China \\ Email: *weipp@163.com
}

How to cite this paper: Wei, F.P., Lan, Z.X., Shen, L.M., Liu, B.R. and Xue, Y. (2017) Comparison of Two Possible Evolutionary Mechanisms of the 62 tRNA Codon Sequences. J. Biomedical Science and Engineering, 10, 172-181.

https://doi.org/10.4236/jbise.2017.104014

Received: March 1, 2017

Accepted: April 27, 2017

Published: April 30, 2017

Copyright (c) 2017 by authors and Scientific Research Publishing Inc. This work is licensed under the Creative Commons Attribution International License (CC BY 4.0).

http://creativecommons.org/licenses/by/4.0/

\begin{abstract}
The tRNAs were divided into 62 groups based on the codons they carried and networks were constructed. Phylogenetic trees were constructed in parallel and antiparallel directions based on the parameters of these networks. Point mutations were found in the codon sites in the same clusters in the entire parallel and antiparallel phylogenetic trees, whereas there was no evidence of a complementary duplication mechanism in the corresponding antiparallel phylogenetic trees. The codons of isoaccepting tRNAs were found in neighboring clusters or distributed within a very small distance so the codons with only one base difference remained very close, which was perfectly consistent with the hypothesis that a new tRNA gene could be recruited from an isoaccepting group via another point mutation.
\end{abstract}

\section{Keywords}

Phylogenetic Trees, Point Mutation, Template Duplication, Network

\section{Introduction}

There are many reports on mitochondrial (mt) tRNA mutations would linked with inherited diseases [1] [2] [3], i.e., mutations in $t R N A^{I l e}, t R N A^{L y s}$ and $t R N A^{S-}$ er $(U C M)$ are known to cause different $\mathrm{mt}$ encephalomyopathies or non-syndromic deafness [3]. The mutation of tRNA genes has a research hotspot. Many tRNA sequences have been found and deposited in a database [4] and they all conform to one of 62 codon sequence groups (two codon groups are absent from the database, i.e., aaa and cta). All modern tRNA sequences have evolved from a common ancestor, but their evolutionary mechanism remains an open question. Two possible mechanisms have been proposed to explain the origin and evolution of modern tRNAs, i.e., "point mutation" and "template duplication" [5]. The "point mutation" mechanism suggests that divergence occurred mainly via 
"mutation" [6] [7] [8] [9] [10]. It is hypothesized that a new sequence may be recruited from the mutant RNA molecule or be recruited from an isoaccepting group by another point mutation in the sequence that concurrently changed the tRNA amino acid identity and its messenger RNA coupling capacity, which is known as "codon capture" or "tRNA gene recruitment" [11]. In contrast to evolution via mutation, the "template duplication" mechanism proposes that tRNAs diverged from a precursor via a duplication mechanism. It is hypothesized that primordial tRNAs with hairpin structures associate themselves with a template and produce another RNA via sequence complementarity [12]-[17]. It is thought that four initial pairs of pre-tRNAs with complementary anti-codons could have been capable of generating all 64 anticodons.

Theoretically, if modern tRNAs evolved via point mutations, the matching scores of sequences that align in a parallel direction, 5' $\rightarrow 3^{\prime}$ vs. $5^{\prime} \rightarrow 3^{\prime}$, should be larger than the scores aligned in an antiparallel direction, 5 ' $\rightarrow 3^{\prime}$ vs. $3^{\prime} \longrightarrow 5$ '. If modern tRNAs evolve by complementary duplication method, however, the opposite would be true. To identify the most likely mechanism for tRNA evolution, we compared tRNA sequences in parallel and antiparallel directions, and we constructed two types of networks to make a comparison. The corresponding parallel and antiparallel phylogenetic trees of the 62 codon sequences could be generated by altering the parameters of these networks using the neighbor-joining method to different degrees. We discuss the evolutionary properties of the codon sequences for the 62 tRNA groups and the two types of phylogenetic trees.

\section{Materials and Methods}

\subsection{Experimental Data}

The 3695 tRNA sequences are acquired from the database (http://trnadb.bioinf.uni-leipzig.de/) [1] and the highly similar species in the database were filtered. Each tRNA sequence contained 76 bases and we removed the bases in the variable stem and the poly-A tail.

\section{2. tRNA Network Construction}

\subsubsection{Important Network Parameters}

Degree and clustering coefficient: the network is made up of a set of nodes (vertices) and the connections between them (edges or links). The features and the nature of the network were indicated by two main parameters [18], i.e., the degree, $\mathrm{k}$, and the clustering coefficient, $\mathrm{c}$, of the nodes. The degree $\mathrm{k}$ of a node was the number of nodes with which it was connected. If a node connected with $i$ other nodes and $j$ edges had connections within these $i$ nodes, the clustering coefficient $c$ of the original node was defined as $C=2 j / i(i-1)$, where $i(i-1) / 2$ is the total number of possible connections among $i$ nodes. The clustering coefficient reflected the relationships among the neighbors of a node and it quantified the inherent tendency of the network to cluster. Investigating the degree and clustering coefficient distribution of the network facilitated the study of the local 
properties among the nodes and their global statistics.

\subsubsection{Method for Constructing the tRNA Network}

The steps used to construct the tRNA network were as follows.

(1) Compare the two tRNA sequences stochastically, ith and jth, in parallel direction and antiparallel directions, and record their degree of similarity, $s_{i j}$ where $s_{i j}$ is defined as the alignment scores for the ith and $j$ th tRNAs (shown in Figure 1)

(2) Consider each tRNA as one node in the network and add a connection if two tRNAs have a degree of similarity, $s$, that is larger than a given degree of similarity $s_{0}$.

After repeating these two steps, an undirected tRNA network can be constructed based on a given degree of similarity. In this study, the 3695 tRNA sequences were divided into 62 groups based on their different tRNAs anticodons. The networks were constructed using different degrees of similarity in parallel and antiparallel directions, and the corresponding average degree $\langle D\rangle$ and average clustering coefficient $\langle C\rangle$ of these networks were recorded.

\subsection{Phylogenetic Tree Construction}

We constructed the tRNA phylogenetic trees suing the neighbor-joining method [19]. To determine the evolutionary relationships among the 62 tRNA codon sequences, we used a novel method to compute the evolutionary distances between different tRNAs groups using two important parameters, $\langle D\rangle$ and $\langle C\rangle$, for different networks of tRNAs, rather than comparing only two sequences. The trees were constructed using the following steps.

(i) We used the ith and $j$ th codon groups to produce larger tRNA groups and used them to construct a network, before calculating the average degree $\langle D(i, j)\rangle$ and the average clustering coefficient $\langle C(i, j)\rangle$ for this larger network.

(ii) The evolutionary distance between any two codon groups was computed using the following equation:

$$
\begin{aligned}
& \text { distance }(i, j)=\left\{\begin{array}{l}
\frac{1}{2}(1-S / L) *(\langle D(i, j)\rangle /(N-1)+\langle C(i, j)\rangle), i \neq j \\
0, i=j
\end{array}\right. \\
& \text { parallel 5' TCAA GACTTCTAAAACTCAGAAATAC -ACGTC CAACTTCA GGACAAGAA GAT 3' } \\
& \text { 5, ATC GGG CATGACTÁGAAÁtCÁtgT-G- CGCA GGTTCGAATCC TGC CGTTCÁC } 3 \text {, } \\
& \text { antiparallel } \\
& \text { 5' tCaA GaCtTctaAaActCagaAataC- ACGTC CAACtTCa GgacaAgaA Gat 3' }
\end{aligned}
$$

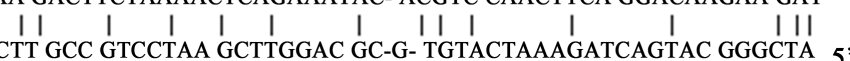

Figure 1. The degree of similarity of two tRNA segments in two directions, parallel: $S=8$, antiparallel: $S=15$. The alignment score was defined as the degree of similarity, i.e., the number of bases in two tRNAs that were the same in a parallel comparison, such as A-A, C-C, T-T, and G-G, or complementary in an antiparallel comparison, such as A-T, C-G, and G-T. 
where $i$ and $j$ ran from 1 to 62 . In this study, $L$ was the length of one tRNA, and $N$ was the total number of nodes in the network. We use $\langle D(i, j)\rangle$ and $\langle C(i, j)\rangle$ to represent the alignment scores of two different codon groups, which showed the evolutionary affiliation of tRNAs with two different codons, which helped us to interpret the overall evolutionary trend of the tRNA sequences to some extent.

(iii) We constructed the phylogenetic tree for the 62 tRNA codon sequences using PHYLIP 3.67 and TreeView 1.6.6.

\section{Results}

Figure 2 shows the diverse relationships among the phylogenetic trees of the 62 tRNA codon groups. Each tree branch represents one group with a specific codon sequence. Figure 2(a) shows that a degree of similarity of $S=10$ produced two large clusters, i.e.,

$(($ att, $($ cct, $($ act,$(\operatorname{tgt},(\operatorname{tga},(\operatorname{cga},($ tat,$(\mathrm{tct},($ gct,,$($ aag:agt $))))))))))$ ), (cgg, $(\mathrm{tca},(\mathrm{gca},(\mathrm{agg},(\mathrm{tcg},(\mathrm{atc},(\mathrm{acc}: \mathrm{acg}))))))), \mathrm{gcc},(\mathrm{gcg},(\mathrm{gtg}: \operatorname{tgg}))$ and $((\operatorname{cgc},($ gta,$($ gtc,$($ gac,$($ aca,$($ ctg,,$($ ggg:ccc $)))))))$, agc, (cca, (acc,(gtt,(aga,(gga,(ctt:ggt))))),ggc,(ctc,(tcc:ttg)), as well as 15 isolated branches and one small cluster in the overall parallel phylogenetic tree, where each large cluster comprised three small clusters. the sequences atg, aac, tta, ata, caa, ttt, cat, gat, gag, tag, gaa, and taa were found in isolated branches, but their evolutionary distance was small. They exhibited a regular ladder ranked appearance. All of the codons in the large clusters supported the point mutation mechanism and each had a matching codon with only one base difference in the same site. We also found that some codons of the isoaccepting tRNAs appeared in the same cluster and neighboring clusters. For example, six codons the amino acid $A r g$, i.e., cct, tct, tcg, acg, ccg, and gcg, for were in the same cluster, three of them were in a neighboring branch, and three were dispersed among different branches. In contrast to the parallel phylogenetic tree for $s=10$, the antiparallel phylogenetic tree for $s=10$ only contained two large clusters and some isolated branches, and the codons of these two large clusters exhibited a regular laddered rank. Thus, these results did not support "template duplication mechanism." Some codons of isoaccepting tRNA sequences also appeared in the same cluster, e.g., four codons for Ser were in the same cluster, whereas the other two codons were in another cluster. Most codons for isoaccepting tRNA sequences were dispersed throughout the tree.

For $s=35$, a comparison of Figure 2(a) and Figure 2(c) showed that the distribution of the parallel phylogenetic tree in Figure 2(c) was clearly different, with fewer isolated branches while the codons in the large cluster were more dispersed. Two small clusters and one large cluster comprised the whole tree, where most codons had matching codons with only one base difference in the small cluster, although a few had no mutated codons in the upstream branch. More codons of the isoaccepting tRNA sequences were present in the same cluster and they were closer. As shown in Figure 2(d), the distribution of the anti- 


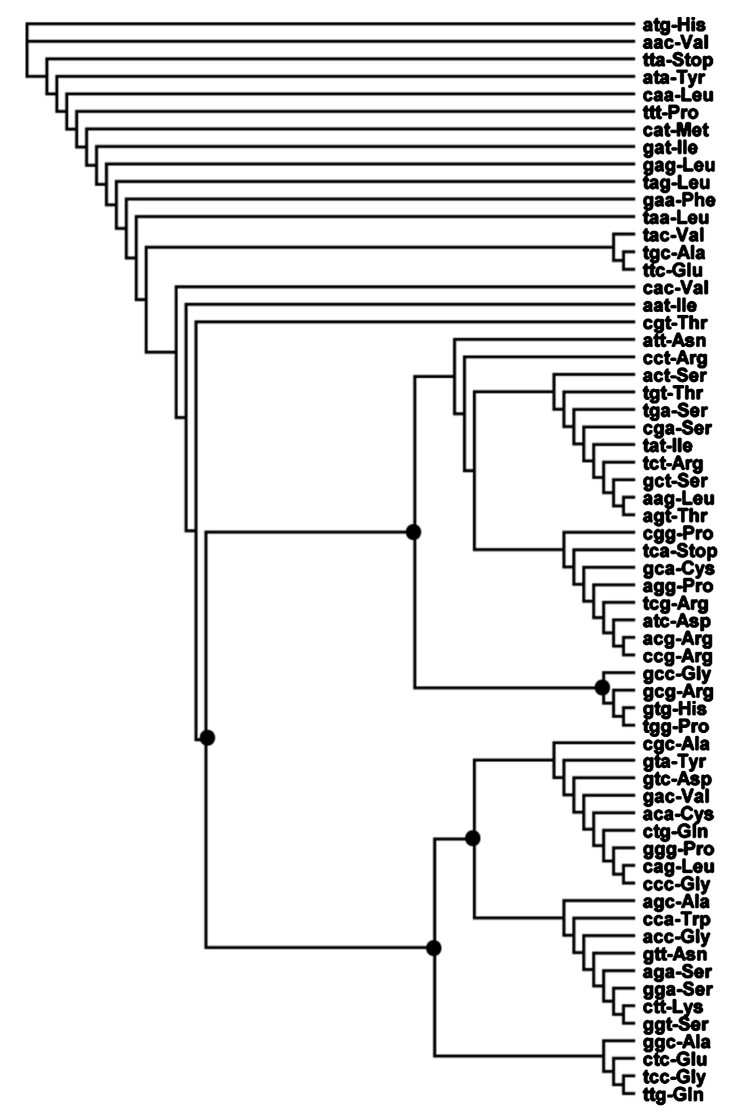

(a)

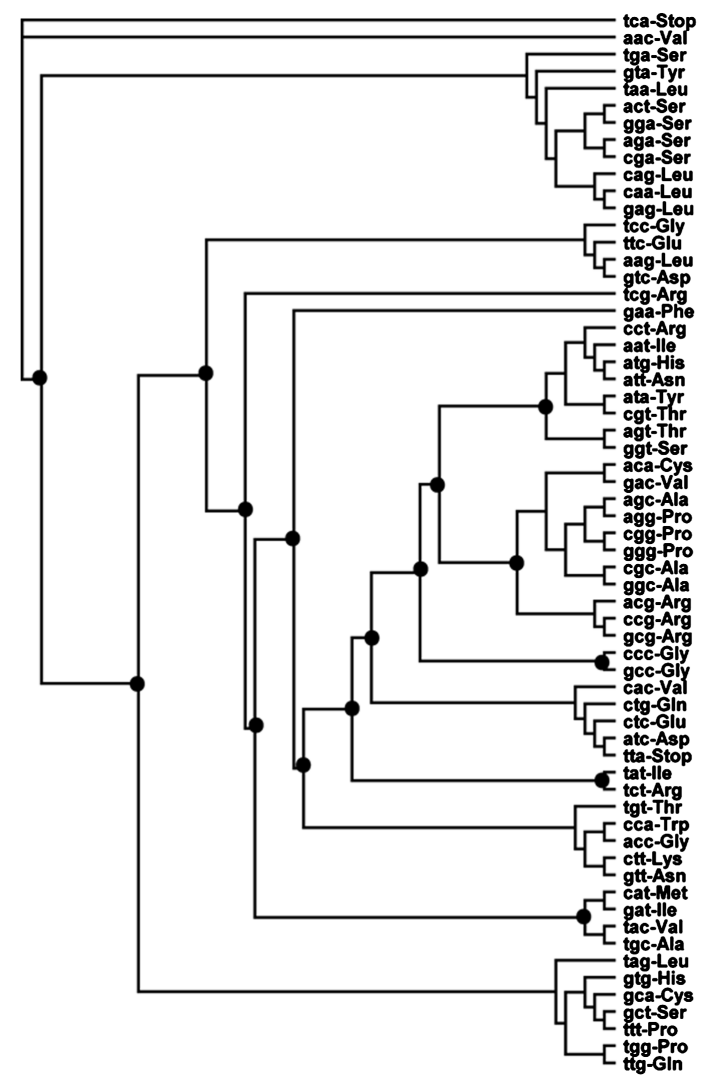

(c)

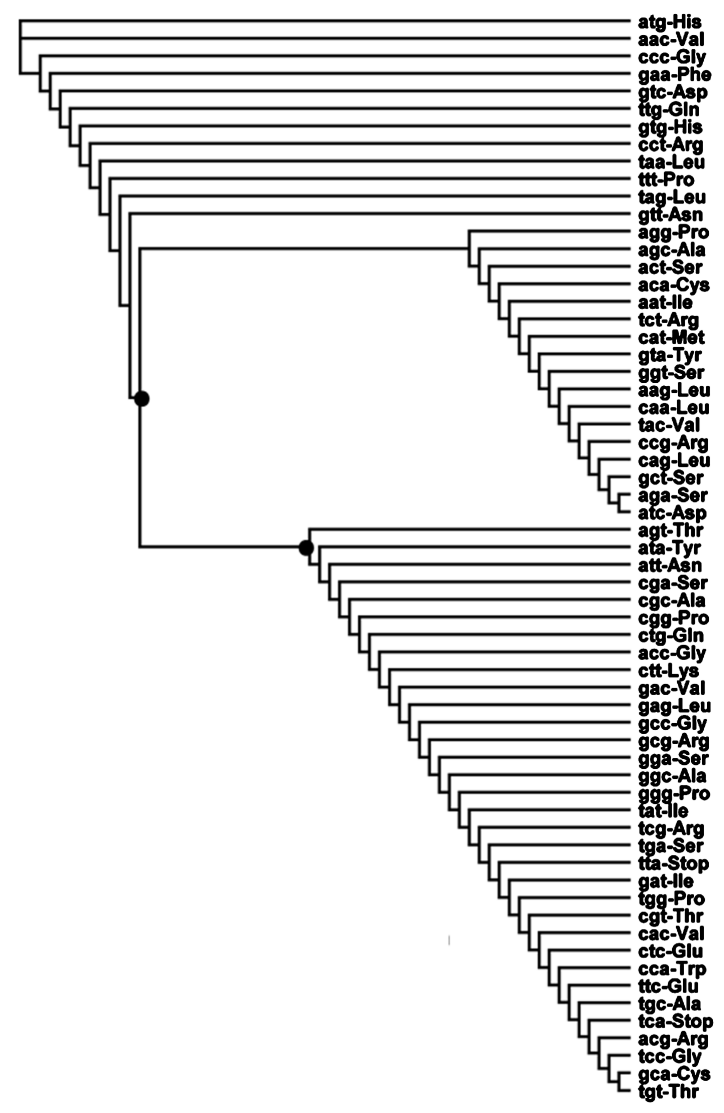

(b)

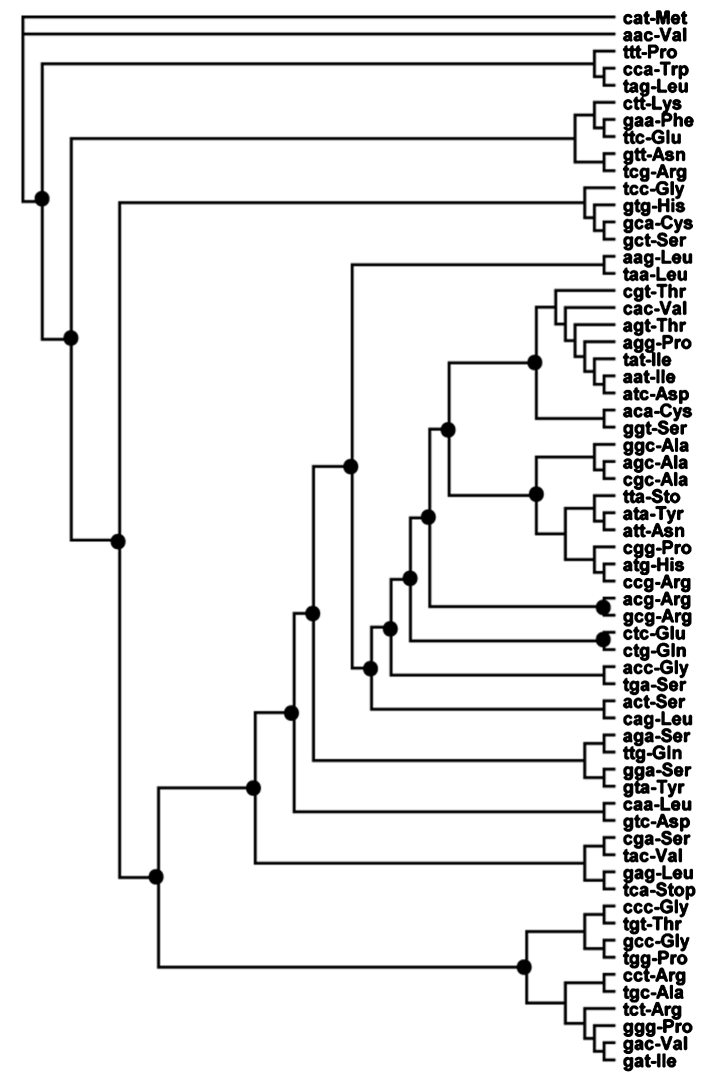

(d) 


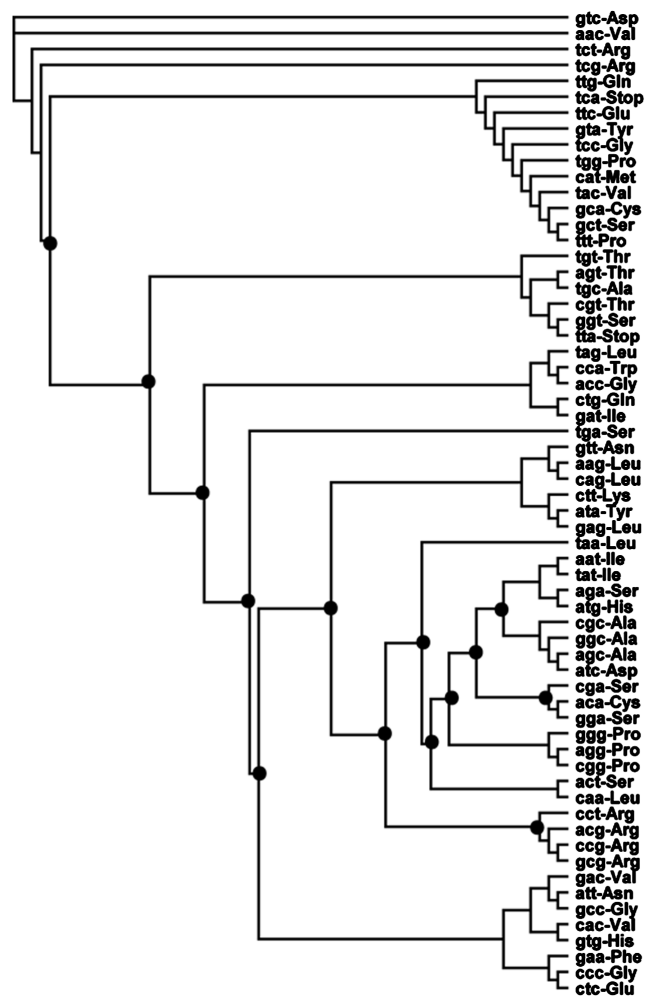

(e)

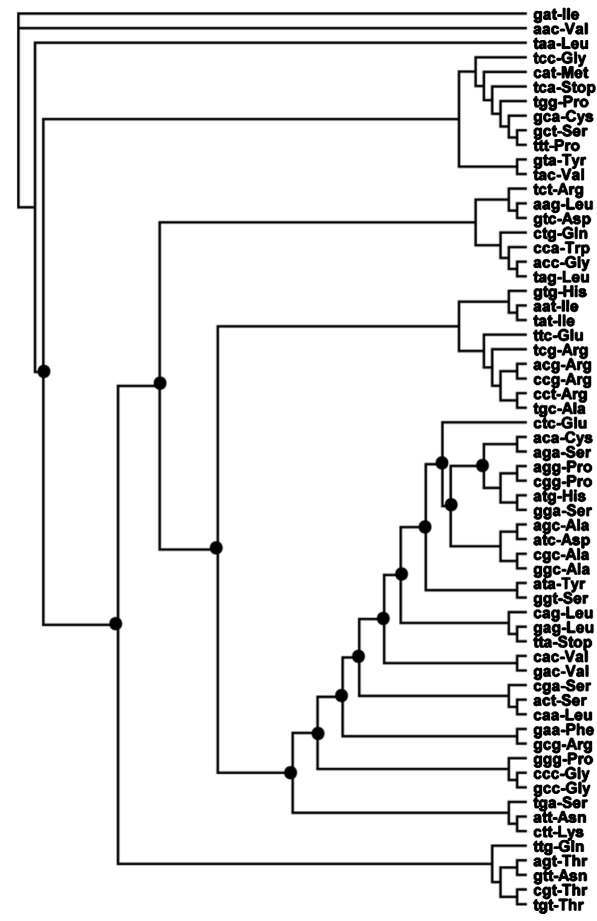

(g)

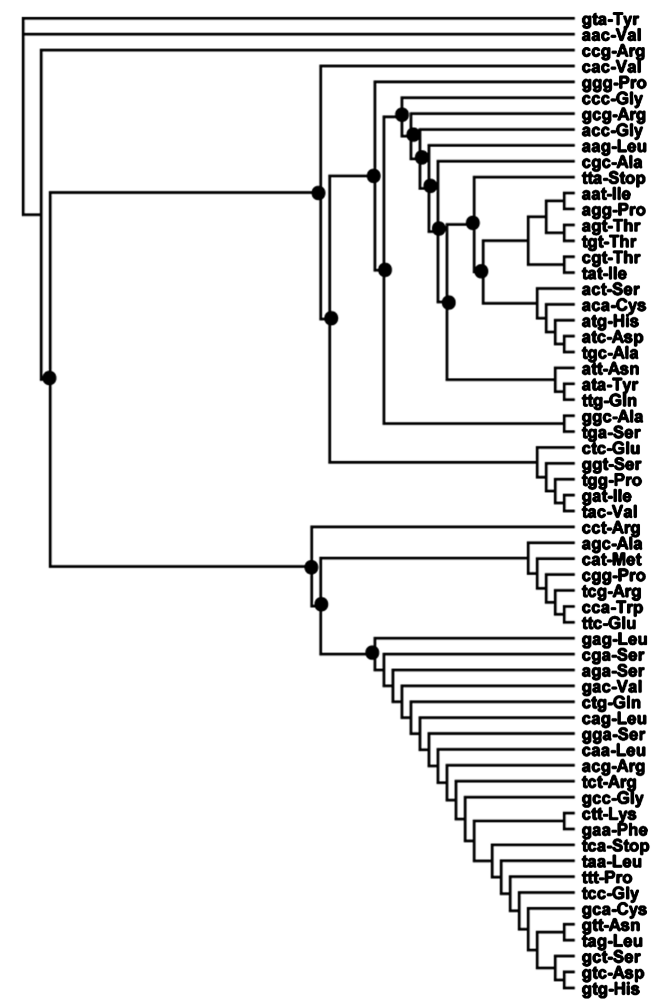

(f)

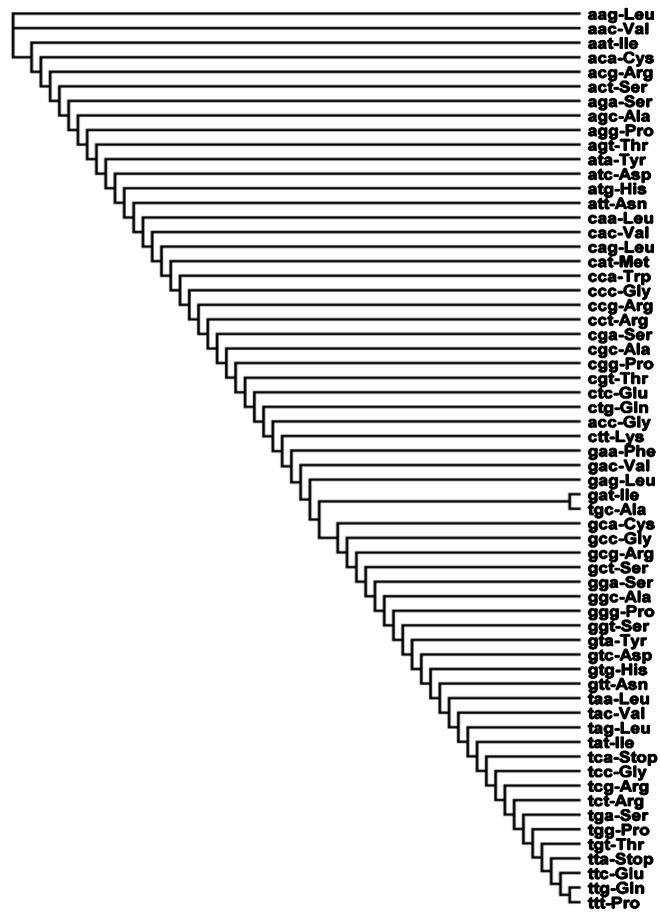

(h)

Figure 2. Phylogenetic trees of 62 cod on sequences with a degree of similarity of $s=10, s=35, s=45$ and $s=50$ for the antiparallel phylogenetic tree and antiparallel phylogenetic tree. Each tree branch represents one group codon and its abbreviated letters. The black solid dots $(\bullet)$ in the trees represent the codons that agree with the point mutation mechanism in a branch. (a) Parallel phylogenetic tree with $s=10$; (b) Antiparallel phylogenetic tree with $s=10$; (c) Parallel phylogenetic tree with $s=35$; (d) Antiparallel phylogenetic tree with $s=35$; (e) Parallel phylogenetic tree with $s=45$; (f) Antiparallel phylogenetic tree with $s=45$; (g) Parallel phylogenetic tree with $s=50$; (h) Antiparallel phylogenetic tree with $s=50$. 
parallel phylogenetic tree with $s=35$ was more compact compared with the parallel phylogenetic tree with $s=35$, i.e., three small clusters, one large cluster, and two isolated branches comprised the entire tree. Two clusters formed the core of the larger cluster. However, it appeared to be more disperse than the antiparallel phylogenetic tree with $s=10$. Surprisingly, most of the codons in the clusters did not have template duplication codons, even though they had copies upstream. Their evolution did not agree with the "template duplication mechanism."

As the degree of similarity $(s) S$ increased, more dispersed clusters appeared in the larger cluster in the parallel phylogenetic tree. As shown in Figure 2(e), the largest cluster is divided into six small clusters with $s=45$, where gtc, aac, tct, and tcg remained in isolated branches. A comparison of Figure 2(a), Figure 2(c), and Figure 2(e) shows that the codon aac occupies a basal clade and it did not vary with the degree of similarity $(s)$ in the three phylogenetic trees. The evolutionary relationships among the branches of the three phylogenetic trees were always ordered and stable with different values of $s$. Most tRNA anticodons in the same clusters had a high match with the "point mutation mechanism" and most had only one base difference from the anticodon sites, which suggests that they evolved by mutating from the same ancestral sequences via point mutations. At the same time, many isoaccepting tRNA codons were distributed in the same cluster and they had a very compact structure. However, not all of the isoaccepting tRNA codons had neighboring relationships, e.g., four codons in the isoaccepting group for $\mathrm{Arg}$ were neighboring whereas the other two were far from their branch, which suggested that these two codons may have evolved from neighboring codons. In contrast to the sparse distribution of the parallel phylogenetic tree, the antiparallel phylogenetic tree with $s=45$ comprised two large clusters with a more compact branch distribution. One large cluster also appeared to have a laddered rank. Similar results are shown in Figure 2(f) where more isoaccepting tRNA codons had neighbors in the same cluster, e.g., five codons for Leu were adjacent in a large cluster. However, not all of the isoaccepting tRNA codons were allocated to the same cluster. Figure 2(f) shows that evidence for duplication was absent from the entire tree.

As the degree of similarity $s$ increased to 50 , the distances of most codon branches in the parallel and antiparallel phylogenetic trees tended toward 1, as shown in Figure 2(g). The parallel phylogenetic trees with $s=50$ displayed a laddered rank, but there were few isolated branches and the distances among most branches were similar. There were also many small clusters that contained some codons of isoaccepting tRNAs. Most of these codons showed no evidence point mutations traces at higher levels in the upstream branch. With $s>50$, most of the distances for the codons in the parallel phylogenetic trees were equal to 1 . There were clear differences in the parallel phylogenetic trees in Figure 2(h), while most of the distances among codon branches in the antiparallel phylogenetic trees with $s=50$ were equal to 1 , and the distribution of the tree was a laddered rank. 


\section{Conclusions}

We constructed $62 \times 61 / 2$ groups of networks and eight phylogenetic trees of tRNAs based on a point mutation mechanism and a complete duplication mechanism. We analyzed the features of each codon branch and the phylogenetic trees in parallel and antiparallel directions. We found that point mutations had an important role in the evolution of the 62 codon sequence groups. Point mutations were found in the codon sites in the same clusters in four complete parallel phylogenetic trees, whereas there was no evidence for a complementary duplication mechanism in the corresponding antiparallel phylogenetic trees. In addition, many codons of isoaccepting tRNA were found in neighboring clusters or very close clusters. The codons with one site difference were very close together. These results support the hypothesis that new tRNA genes were recruited from one isoacceptor group to another via a point mutation in the sequence.

Surprisingly, most of the codons in the antiparallel phylogenetic trees in the same cluster did not have complementary copies without the point mutation codons, although they were compared in the antiparallel direction. As shown in Figure 3, most codons in the antiparallel phylogenetic tree with $s=45$ had no

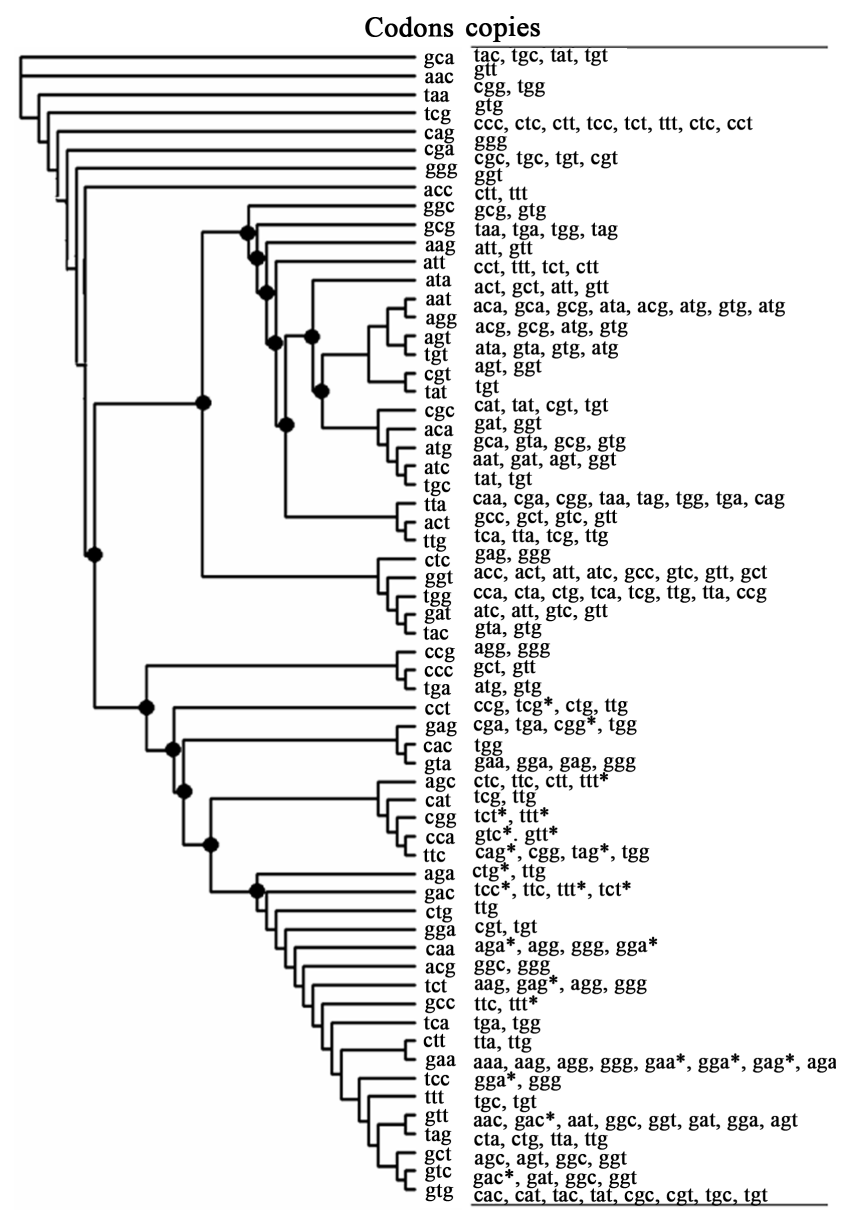

Figure 3. Codons in the antiparallel phylogenetic tree where $s=45$ and its possible complementary duplication anticodons. The symbol “»” indicates that this anticodon has a copy in the cluster. 
complementary codons, although many had point mutation codons in the same cluster. These observations also applied to the other antiparallel trees with different $s$ values. They are marked with a black solid $\operatorname{dot}(\bullet)$ in all of the trees and they provide powerful support for the role of point mutations in the evolution of the 64 tRNA codon sequences.

Point mutations occur in the mitochondria tRNA; some positions will cause loss of stability or produce disease. As more and more tRNA sequencing, the mutation of the tRNA gene sequences is studied further, and the evolutionary mechanisms of the RNA will be proven.

\section{Acknowledgements}

This paper was supported by the National Science Foundation of Guanxi of China (Grant No.: AE052095) and the National Regional Fund (Grant No.: 11262003) and Guangxi Key Laboratory for the Relativistic Astrophysics.

\section{References}

[1] Scaglia, F. and Wong, L.-J.C. (2008) Human Mitochondrial Transfer RNAs: Role of Pathogenic Mutation. Disease Muscle Nerve, 37, 150-171. https://doi.org/10.1002/mus.20917

[2] Suzuki, T., Nagao, A. and Suzuki, T. (2011) Human Mitochondrial tRNAs: Biogenesis, Function, Structural Aspects, and Diseases. Annual Review of Genetics, 45, 299 329. https://doi.org/10.1146/annurev-genet-110410-132531

[3] Da Pozzo, P., Cardaioli, E., Malfatti, E., et al. (2009) A Novel Mutation in the Mitochondrial tRNA Pro Gene Associated with Late-Onset Ataxia, Retinitis Pigmentosa, Deafness, Leukoencephalopathy and Complex I Deficiency. European Journal of Human Genetics, 17, 1092-1096. https://doi.org/10.1038/ejhg.2009.12

[4] Jühling, F., Mörl, M., Hartmann, R.K., et al. (2009) tRNAdb 2009: Compilation of tRNA Sequences and tRNA Genes. Nucleic Acids Research, 37, D159-D162. https://doi.org/10.1093/nar/gkn772

[5] Wei, F., Li, S. and Ma, H. (2006) Comparing Two Evolutionary Mechanisms of Modern tRNAs. Molecular Phylogenetics and Evolution, 38, 1-11.

[6] Eigen, M. and Winkler, O.R. (1981) Transfer-RNA: The Early Adaptor. Naturwissenschaften, 68, 217-228. https://doi.org/10.1007/BF01047323

[7] Eigen, M. and Winkler, O.R. (1981) Transfer-RNA, an Early Gene? Naturwissenschaften, 68, 282-292. https://doi.org/10.1007/BF01047470

[8] Osawa, S. and Jukes, T.H. (1989) Codon Reassignment (Codon Capture) in Evolution. Journal of Molecular Evolution, 28, 271-278. https://doi.org/10.1007/BF02103422

[9] Osawa, S., Collins, D., Ohama, T., Jukes, et al. (1990) Evolution of the Mitochondrial Genetic Code. III. Reassignment of CTN Codons from Leucine to Threonine during Evolution of Yeast Mitochondria. Journal of Molecular Evolution, 30, 322 328. https://doi.org/10.1007/BF02101886

[10] Osawa, S., Jukes, T.H., Watanabe, K. and Muto, A. (1992) Recent Evidence for Evolution of the Genetic Code. Microbiological Reviews, 5, 229-264.

[11] Margaret, E.S., et al. (1998) Evolution of a Transfer RNA Gene through a Point Mutation. Anticodon, 279, 1665-1670.

[12] Kuhn, H. and Waser, J. (1982) Evolution of Early Mechanisms of Translation of Ge- 
netic Information into Polypeptides. Nature, 298, 585-586. https://doi.org/10.1038/298585a0

[13] Di, G.M. (1992) On the Origin of the Transfer RNA Molecule. Journal of Theoretical Biology, 159, 199-214.

[14] Manfred, E. and Peter, S. (1979) The Hypercycle-A Principle of Natural Self-Organization. Springer-Verlag, Berlin.

[15] Peter, S. (1993) RNA Based Evolutionary Optimization. Origins of Life, 23, 373-391.

[16] Biebricher, C., Eigen, M. and Mccaskill, J. (1993) Template-Directed and TemplateFree RNA Synthesis by Q-Beta Replicase. Journal of Molecular Biology, 231, 175179. https://doi.org/10.1006/jmbi.1993.1271

[17] Sergey, R., et al. (1993) Transfer RNAs with Complementary Anticodons: Could They Reflect Early Evolution of Discriminative Genetic Code Adaptors? Proceedings of the National Academy of Sciences, 90, 4723-4727. https://doi.org/10.1073/pnas.90.10.4723

[18] Barabási, A. and Albert, R. (1999) Emergence of Scaling in Random Networks. Science, 286, 509-512. https://doi.org/10.1126/science.286.5439.509

[19] Saitou, N. and Nei, M. (1987) The Neighbor Joining Method: A New Method for Reconstructing Phylogenetic Trees. Molecular Biology and Evolution, 4, 406-425.

Submit or recommend next manuscript to SCIRP and we will provide best service for you:

Accepting pre-submission inquiries through Email, Facebook, LinkedIn, Twitter, etc. A wide selection of journals (inclusive of 9 subjects, more than 200 journals) Providing 24-hour high-quality service User-friendly online submission system Fair and swift peer-review system Efficient typesetting and proofreading procedure Display of the result of downloads and visits, as well as the number of cited articles Maximum dissemination of your research work

Submit your manuscript at: http://papersubmission.scirp.org/

Or contact jbise@scirp.org 